\title{
Web of Conferences : Spécifications et limite d'acceptation en incertitude de mesure pour les moyens de contrôle en atelier, dans les usines mécaniques PSA : Témoignage industriel.
}

\author{
Jacques Hurel
}

PSA Peugeot Citroën Automobiles, DRD/DPMO/CIMB/CIUO/ITPU/UPQM, 18 rue des Fauvelles 92256 La GarenneColombes Cedex, France

\begin{abstract}
Résumé. Pour la production en interne de pièces de moteurs ou de boîtes de vitesses, notamment, PSA utilise un critère de capabilisation pour ses moyens de mesure appelé CMC, cette démarche de capabilisation est décrite dans la norme CNOMO E41 $36110 \mathrm{~N}$. Plus généralement, par extension, l'incertitude globale de mesure tolérée, se situe à IT/8. La principale difficulté, dans l'évaluation de cette incertitude de mesure, réside dans l'obtention de valeurs de référence fiables, sur des étalons ou des pièces, avec un minimum d'erreurs pour qualifier les moyens de mesure. Il est proposé ici de présenter ces difficultés et aussi les orientations afin de réduire les écarts de justesse.
\end{abstract}

\begin{abstract}
For in-house production of parts of engines or gear boxes, in particular, PSA uses a criterion of capabilisation for its means of measurement called $\mathrm{CMC}$, this approach of capabilisation is described in standard CNOMO E41 36,110 NR. More generally, by extension, the total uncertainty of tolerated measurement, is located at IT/8. The main difficulty, in the evaluation of this uncertainty of measurement, lies in obtaining reliable values of reference, on standards or parts, with a minimum of errors to qualify the means of measurement. It is proposed here to present these difficulties and also the orientations in order to reduce the variations of accuracy.
\end{abstract}

\section{Rappels sur le critère de capabilité CMC pour les moyens de mesure (Norme CNOMO)}

Cette démarche est largement appliquée pour des moyens de mesure situés au pied des machines d'usinage, dits en Bord de Ligne.

Démarche: après une phase préliminaire de capabilisation, qui permet d'évaluer la répétabilité du moyen avec un étalon, puis la répétabilité avec une pièce, la seconde phase consiste à évaluer, à la fois une répétabilité de mesure, mais aussi une justesse de ces mesures par rapport à un jeu de 5 pièces préalablement mesurées en Métrologie.
Rappel des modes de calcul des critères d'acceptation du CMC :

Phase préliminaire :

- Incertitude (Répétabilité) sur l'étalon, Ie $=2 * \sigma e$ $\sigma \mathrm{e}=$ racine carrée de la variance sur 5 ou 10 mesures (Ve). Avec Ie $\leq \mathrm{IT} / 20$ - Incertitude (Répétabilité) sur une pièce, $\mathrm{Ir}=2 * \sigma r$ $\sigma \mathrm{r}=$ racine carrée de variance sur 10 mesures $(\mathrm{Vr})$. Avec Ir $\leq \mathrm{IT} / 8$

Phase d'agrément, sur 5 pièces avec 5 mesures par pièce :

- Incertitude globale $\operatorname{Ig}=|\mathrm{J}|+2 *$ racine carrée de la somme des variances $(\mathrm{Vg}+\mathrm{Ve})$. Avec $\mathrm{J}=$ moyenne des écarts par rapport aux valeurs de référence, sur les 25 mesures et $\mathrm{Vg}$ la variance sur ces 25 mesures.

Le $\mathrm{CMC}=\mathrm{IT} /(2 * \mathrm{Ig}) \quad$ Avec $\mathrm{CMC} \geq 4$ sur IT $>16 \mu$ 


\section{Impact du niveau de capabilité d'un moyen de mesure sur l'évaluation de la capabilité d'un process de production.}

Ce sont les écarts de justesse induits par les moyens de mesure qui sont le plus impactant sur le résultat des évaluations des capabilités des process de production.

Ces erreurs induites par le défaut de justesse des moyens de mesure, tout en étant dans une limite acceptable, sur des critères de capabilité process, comme le CMK, peuvent s'avérer très importantes. De manière moindre les erreurs de répétabilité, peuvent aussi influencer les résultats de capabilité des process de production.

A titre d'exemple, voici avec un CMC de 5, suivant deux types d'erreurs sur le moyen de mesure, les écarts qui peuvent être constatés, au pire des cas, sur l'évaluation d'un critère CMK qui se trouve théoriquement à 1,43 , avec un décentrage de la production qui est à IT/6 :

$-1^{\circ}$ ) avec une erreur de justesse importante et une bonne répétabilité du moyen de mesure, le CMK pourra varier de $\pm 25 \%$, suivant le sens plus ou moins favorable de cette erreur de justesse du moyen de contrôle.

$-2^{\circ}$ ) avec une faible erreur de justesse et une forte erreur en répétabilité du moyen de mesure, le CMK sera minimisé de l'ordre de $13 \%$.

Ceci montre, encore une fois ce sont les erreurs de justesse du moyen de mesure qui sont, et de loin, les plus pénalisantes sur l'évaluation du critère de capabilité process de type CMK.

\section{Difficultés d'obtention pour des CMC conformes.}

Indépendamment des inadéquations du moyen de mesure, dans sa conception, qui peuvent se relever en phase de capabilisation pour la caractéristique à mesurer, c'est habituellement sur la mesure des 5 pièces pour l'agrément capabilité du moyen de mesure que nous rencontrons des difficultés. Nous relevons des écarts non fondés, si des précautions n'ont pas été prises lors des mesures en Métrologie de ces 5 pièces, mesures et précautions qui sont adaptées au moyen à évaluer. Ces écarts, parfois trop importants, ne nous permettent pas de statuer sur la capacité du moyen à pouvoir mesurer les caractéristiques, produit-process, pour lesquelles il a été conçu.

\section{Les précautions à mettre en œuvre, sur la mesure des 5 pièces pour agrément, en Métrologie.}

Outre l'importance de l'emplacement des points de mesure pris en compte par rapport au moyen de mesure à évaluer, avec la prise en compte des défauts induits par les valeurs d'états de surface, pour lesquels il faut parfois apporter des correctifs aux valeurs relevées, ou de toutes les autres sources d'erreurs (linéarité, amplification, ...), ce sont le manque de précision ou le manque de résolution dans les moyens de référence utilisés pour mesurer les étalons ou les 5 pièces d'agrément, qui peuvent être à l'origine des écarts observés le plus souvent lors des capabilisations des moyens de mesure en phase d'agrément.

Ces remarques sont d'autant plus importantes que les IT à mesurer sont réduits, notamment sur des IT de $16 \mu$ à $30 \mu$.

\section{Les principales composantes en jeu dans pour la maitrise de l'incertitude de mesure pour un $\mathrm{CMC}$ viable.}

\begin{tabular}{|l|}
\hline Résolution sur l'instrument de mesure en Métrologie pour étalon \\
\hline $\begin{array}{l}\text { Incertitude en Métrologique sur la valeur donnée sur l'étalon } \\
\text { (Justesse - Répétabilité) \& Cote gravée arrondie }\end{array}$ \\
\hline $\begin{array}{l}\text { Incertitude sur la valeur prise en référence sur l'étalon pour } \\
\text { l'instrument de mesure à évaluer (Justesse - Répétabilité) }\end{array}$ \\
\hline Résolution sur l'instrument de mesure à évaluer \\
\hline $\begin{array}{l}\text { Résolution sur l'instrument de mesure en Métrologie pour les } \\
\text { mesures des 5 pièces de la phase d'agrément }\end{array}$ \\
\hline $\begin{array}{l}\text { Incertitude en Métrologie sur la valeur prise en référence pour } \\
\text { les } 5 \text { pièces de la phase d'agrément (Justesse - Répétabilité) }\end{array}$ \\
\hline $\begin{array}{l}\text { Répétabilité propre au moyen de mesure à évaluer avec les } 5 \\
\text { pièces en phase d'agrément }\end{array}$ \\
\hline $\begin{array}{l}\text { Erreur de justesse propre au moyen de mesure à évaluer avec } \\
\text { les } 5 \text { pièces en phase d'agrément }\end{array}$ \\
\hline
\end{tabular}

\section{Les résolutions et les incertitudes des moyens de référence à utiliser en Métrologie pour la mesure des étalons ou des 5 pièces d'agrément.}

Les recommandations que nous trouvons dans nos procédures actuelles mentionnent les valeurs suivantes, pour un IT à mesurer qui soit supérieur à $16 \mu$ :

- Résolution moyen de mesure à évaluer à IT/20.

- Incertitude du moyen de référence en Métrologie à IT/16

Ces valeurs ne sont pas compatibles avec le besoin pour évaluer les côtes des étalons ou des 5 pièces d'agrément.

Sur des IT compris entre $16 \mu$ et $30 \mu$, voire $50 \mu$, pour ne pas se mettre en situation d'avoir des écarts entre les valeurs données sur les étalons et les valeurs données sur les 5 pièces d'agrément par le moyen à évaluer, il faut se rapprocher, autant que possible, des valeurs suivantes :

- Avoir 4 décimales sur l'affichage du moyen de mesure à évaluer en phase de capabilisation (à $0,1 \mu$ près).

- Avoir aussi une résolution, lors de la mesure de l'étalon ou des 5 pièces d'agrément en métrologie de $0,1 \mu$.

- L'incertitude des mesures en Métrologie ne doit pas excéder, dans tous les cas, les $0,5 \mu$. 


\section{Limites technologiques et précision des moyens disponibles pour réaliser les mesures en Métrologie.}

Ces exigences de précision en Métrologie ne peuvent pas toujours être respectées car nous n'avons pas toujours les moyens adaptés qui soient disponibles.

\section{Contraintes Produits à venir.}

De plus en plus souvent, les intervalles de tolérance se réduisent sur les pièces en production pour des moteurs ou des boîtes de vitesses, et nous aurons probablement de plus en plus de difficultés à qualifier nos moyens de mesure si nous ne savons pas améliorer ces précisions de mesure en Métrologie, sur des étalons et des pièces d'agrément.

\section{Conclusions.}

Malgré les difficultés énoncées, nous avons toujours pu résoudre les problèmes rencontrés pour capabiliser et vérifier la qualité de nos moyens de mesure, avec un niveau de précision suffisant, en fonction des IT à mesurer. Mais cela a été parfois au prix de beaucoup de remesure sur ces étalons ou ces 5 pièces d'agrément CMC.

Il nous reste aussi à documenter, à évaluer et à comparer notre processus $\mathrm{CMC}$ avec d'autres stratégies pour la qualification des moyens de mesure, tel le R\&R + justesse, ou l'utilisation de critères de type Cg-Cgk. Et toujours sans perdre de vue l'importance de la justesse. 